\title{
SUMMARIZING AND VISUALIZING WEB PEOPLE SEARCH RESULTS
}

\section{Harumi Murakami, Hiroshi Ueda, Shin’ichi Kataoka, Yuya Takamori, Shoji Tatsumi}

\begin{tabular}{|c|l|}
\hline Citation & $\begin{array}{l}\text { Proceedings of the 2nd International Conference on Agents and Artificial Intelligence - } \\
\text { Volume 1: ICAART, 640-643. }\end{array}$ \\
\hline Proceeding & January 22-24, 2010, in Valencia, Spain \\
\hline Type & Conference paper \\
\hline Textversion & Publisher \\
\hline Rights & $\begin{array}{l}\text { This work is licensed under a Creative Commons } \\
\text { Attribution-NonCommercial-NoDerivatives } 4.0 \text { International License. } \\
\text { https://creativecommons.org/licenses/by-nc-nd/4.0/. }\end{array}$ \\
\hline DOI & $10.5220 / 0002760706400643$ \\
\hline
\end{tabular}

Self-Archiving by Author(s)

Placed on: Osaka City University

Murakami H.; Ueda H.; Kataoka S.; Takamori Y.; Tatsumi S. and (2010). SUMMARIZING AND VISUALIZING WEB PEOPLE SEARCH RESULTS. In Proceedings of the 2nd International Conference on Agents and Artificial Intelligence - Volume 1: ICAART, ISBN 978-989-674-021-4, pages 640-643. DOI: 10.5220/0002760706400643 


\title{
SUMMARIZING AND VISUALIZING WEB PEOPLE SEARCH RESULTS
}

\author{
Harumi Murakami ${ }^{1}$, Hiroshi Ueda ${ }^{2}$, Shin'ichi Kataoka ${ }^{1}$, Yuya Takamori ${ }^{1}$ and Shoji Tatsumi ${ }^{2}$ \\ ${ }^{1}$ Graduate School for Creative Cities, Osaka City University, Japan \\ ${ }^{2}$ Graduate School of Engineering, Osaka City University, Japan \\ harumi@media.osaka-cu.ac.jp,d06tb001@ex.media.osaka-cu.ac.jp,gorennrakusaki@yahoo.co.jp \\ highdefensive@yahoo.co.jp,tatsumi@info.eng.osaka-cu.ac.jp
}

Keywords: Web people search, Summarization, Visualization, Interface.

\begin{abstract}
People search is one major search activity on the Web. If the list of people search results is merely "person 1 , person $2, \ldots$ and so on," users have difficulty determining which person clusters they should select. In this paper, we present a project that summarizes and visualizes Web people search results to help users select person clusters more easily. We explore three ways of summarizing people: (a) selecting terms from the extracted information, (b) combining the extracted information, and (c) obtaining information from external databases referring to the extracted information. To visualize people, we present three types of interfaces: (a) tables, (b) two-dimensional space, and (c) map interfaces. We report the two results of the project. (1) We investigated algorithms for distinguishing individuals with identical names and three ways of summarizing people: extracting keywords, prefectures and vocations; combining vocation-related information; and obtaining locations. (2) We developed prototypes to display separated individuals by three types of interfaces.
\end{abstract}

\section{INTRODUCTION}

People search is one major search activity on the Web. According to (Guha and Garg, 2004), 30\% of queries in Web searches include person names. Person name disambiguation, or distinguishing people with identical names, is becoming more and more important in Web searches. If the list of search results is merely "person 1 , person $2, \ldots$, and so on," users have difficulty determining which person clusters they should select.

This research helps users select person clusters that are separated into different people from the results of person searches on the Web.

Below, in Section 2 we explain the overview of our project and describe the developed algorithms and prototypes in Sections 3-6. Related work is discussed in Section 7.

\section{OVERVIEW OF THE PROJECT}

The aim of this project is to develop interfaces to select and understand people on the Web.
First, we create person clusters by distinguishing individuals from Web people search results. Second, we present interfaces to help users select and understand people.

The main feature of this research is summarizing people. We extract various kinds of attribute information related to people. Instead of displaying all extracted information like traditional information extraction research, we present one representative piece of information by referring to the extracted information.

For example for the first author, from the following list, which is the one representative piece of information?: faculty staff, researcher, professor, Osaka City University professor, or computer scientist? We explore three ways to summarize people: (a) selecting terms from the extracted information, (b) combining the extracted information, and (c) obtaining information from external databases that refer to the extracted information.

We present three types of interfaces to visualize people: (a) tables, (b) two-dimensional space in which similar individuals are displayed close together, and (c) map interfaces. See Figure 1. 
(a) Table interface

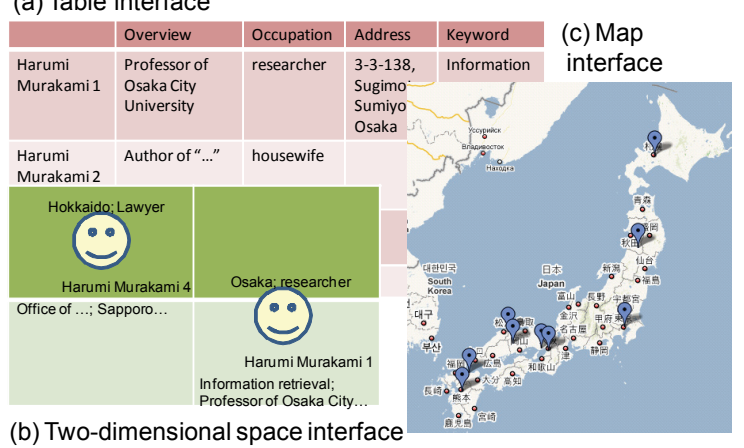

Figure 1: Project overview.

\section{INITIAL PROTOTYPES}

We developed initial prototypes of a table and a twodimensional-space interface (Ueda et al. 2007) that separate individuals from Google people search results and automatically extract attribute information (keywords, prefectures, and vocations).

The table interface displays a list of people with attribute information (Figure 2), and the twodimensional-space interface displays person icons with part of the extracted attribute information (Figure 3).

\subsection{Algorithms}

The algorithms that extract keywords, prefectures, and vocations are described here. They are based on the type of summarization: (a) selecting terms from the extracted information.

\subsubsection{Extracting Keywords}

We extracted nouns near person names using morphological analysis, collocated, and ranked them with TermExtract. For example, for the first author, information retrieval was the top ranked keyword.

\subsubsection{Extracting Prefectures}

We extracted prefecture names by pattern-matching using prefecture dictionaries from texts near the person names. One of the most frequent prefectures is displayed. For example, for the first author, Osaka Prefecture was the answer.

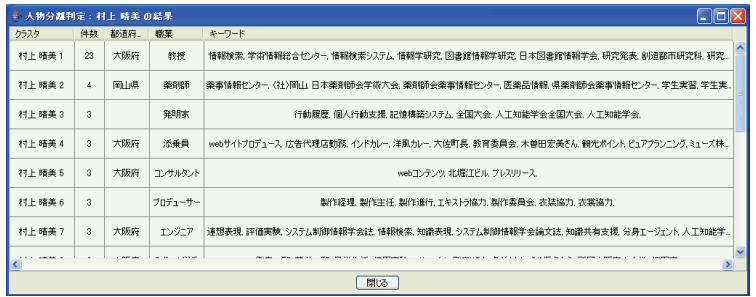

Figure 2: Table interface.

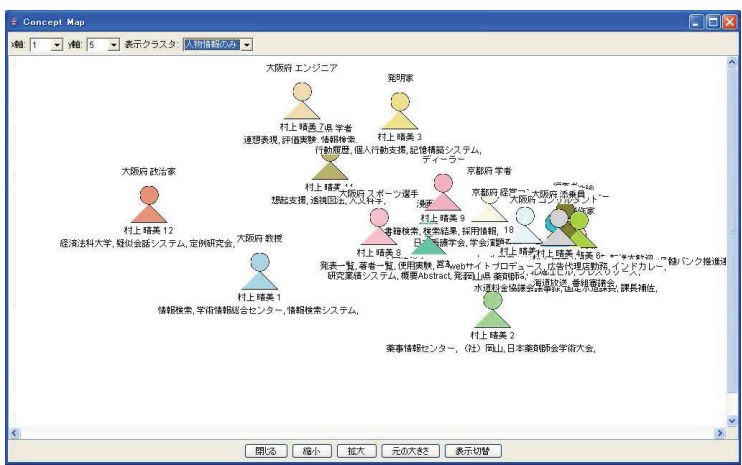

Figure 3: Two-dimensional space interface.

\subsubsection{Extracting Vocations}

We extracted vocations by pattern-matching using dictionaries from texts near the person names. The vocation dictionaries were created from Wikipedia. One of the most frequent vocations is displayed. For example, for the first author, professor was the answer.

Preliminary experiments suggested that vocations and keywords were useful to select people.

\section{DISTINGUISHING DIFFERENT PEOPLE}

We investigated two approaches to separate different people: non-hierarchical clustering and hierarchical clustering.

First in the initial prototype, to simulate how people separate Web pages, non-hierarchical clustering (single path method) was examined. We checked Web pages from the top of the search results and added similar pages to the existing clusters. Since this approach produced too many clusters (Ueda et al. 2007), we need to group them in the future.

As an alternative, we examined a two-step clustering method using person names to distinguish individuals (Kataoka et al. 2008). The key idea was to use person names to create initial clusters by 
combining mechanisms. Our method was comprised of two processes: (1) first step clustering based on the co-occurrence of person names and (2) second step clustering based on hierarchical clustering using keywords. Overall, our method outperformed two baselines and was ranked 2nd in the WePS (Artiles et al. 2007) systems.

In seeking state-of-the art person name disambiguation, since we have found it difficult to automatically distinguish different people, we decided to abandon person name disambiguation and concentrate on summarizing and visualizing manually separated person clusters. In the following sections, we investigate two important bits of attribute information: vocations (Section 5) and locations (Section 6).

\section{ASSIGNING VOCATION-RELATED INFORMATION}

Although vocations are the most informative ways to select people, defining vocations and obtaining good vocation dictionaries was difficult (Ueda et al. 2007). We therefore proposed a method to extract vocationrelated information (VRI) from Web pages in person clusters. VRI is information related to vocation, which is more useful for identifying people (Ueda et al. 2009). We selected three types of VRI: (1) vocation, (2) organization and position, and (3) publication title and role.

The method is comprised of two processes: (1) extraction of VRI candidates using HTML structure and heuristics, and (2) VRI generation using term frequency, clustering synonyms, and calculation using a Web search engine. The method is based on the type of summarization: (b) combining the extracted information.

For example, two pieces of extracted information, Tozai newspaper and Cultural Affairs Department journalist, are combined, and Cultural Affairs Department journalist, Tozai newspaper is generated as a VRI.

The main advantage of our method is to label VRIs to person clusters based on the context, not vocations based on term frequencies.

VRI can be used as one bit of attribute information (e.g., vocations, titles, occupations, or companies) and as one good source of an overview of people.

\section{ASSIGNING LOCATION INFORMATION ON A MAP}

This research (Murakami et al. 2009) aims to display person icons on a map to help users select person clusters that are separated into different people from the results of person searches on the Web. We proposed a method to assign person clusters with one piece of location information.

Our method is comprised of two processes: (1) extracting location candidates from Web pages and (2) assigning location information using a local search engine. The method is based on the type of summarization: (c) obtaining information from external database referring to the extracted information.

For example, the location information of Arise Campus, Kobe Gakuin University, is obtained from Yahoo! Local search using one of the extracted terms: Kobe Gakuin University.

Our main idea exploits search engine rankings and character distance to obtain good location information among location candidates.

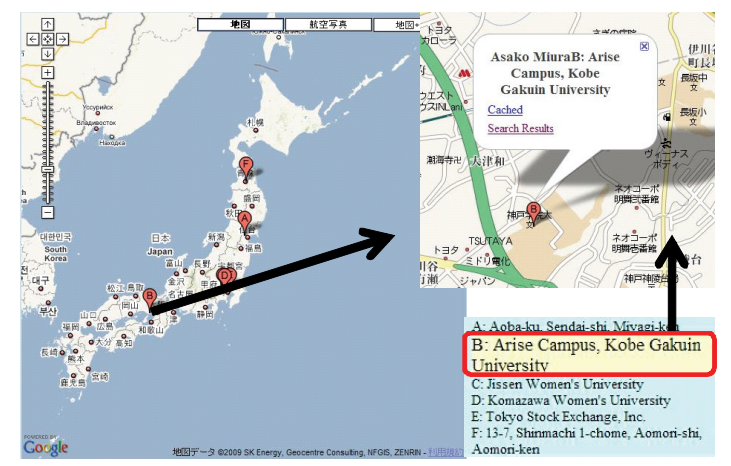

Figure 4: Map interface prototype system.

Figure 4 shows the prototype interface. When a person name is input as a query, Web pages are classified into person clusters, and individual icons that express appropriate locations are displayed on a map. Users can select icons to display their location information to access searched Web pages.

Experimental results revealed the usefulness of our proposed method.

\section{RELATED WORK}

Most people search services (e.g., MyLife) provide manually constructed database input by users or operators. When a user types a person name as a query, a list or table is displayed to select a person. 
Person name disambiguation is manually solved. Ages, locations (usually states in US), titles, and companies are often displayed as attribute information.

Some services and research automatically execute Web people search and display a list or a table. Zoominfo extracts and displays such attribute information as titles and companies. Wan et al. (2005) also separated Web people search results and assigned titles to person clusters to provide a list interface to select people.

We present three kinds of interfaces and explore three types of summarization methods.

Even though much work (Artiles et al., 2007) separates Web pages into person clusters, it seldom assigns labels to person clusters. The WWW2009 WePS-2 workshop evaluated a technique to extract attribute information. Although it aims to extract all attribute information related to people, we assign representative attribute information to person clusters and call this summarization.

Concerning the visualization of people search, Matsuo et al. (2006) visualizes a human network to select people. Mori et al. (2008) presents an interface design for people search that includes a human network and two-dimensional space. Our work is different; we concentrate on summarizing people.

We showed a scenario for people search by names; however, our proposal can be applied to other types of people search such as by keywords. For example, Bill Clinton, George W. Bush, and Barack Obama are summarized and visualized by an input query: president of US.

\section{CONCLUSIONS}

We presented a project that summarizes and visualizes Web people search results to help users select person clusters more easily. We explored three ways to summarize people: (a) selecting terms from the extracted information, (b) combining the extracted information, and (c) obtaining information from external databases referring to the extracted information. We present three types of interfaces to visualize people: (a) tables, (b) two-dimensional space, and (c) map interfaces. We reported the project results.

\section{REFERENCES}

Guha, V., and Garg, A., 2004. Disambiguating People in Search. Stanford University.

Ueda, H., Murakami, H., and Tatsumi, S., 2007. A system that distinguishes different people with identical names on the Web and displays a list by attribute information. In The 21st Annual Conference of the Japanese Society for Artificial Intelligence. (CDROM).

Kataoka, S., Ueda, H., Murakami, H., and Tatsumi, S., 2008. Two-step Clustering based on Person Names to Identify Different People with Identical Names on the Web. In The 22nd Annual Conference of the Japanese Society for Artificial Intelligence. (CD-ROM).

Artiles, J., Gonzalo, J., and Sekine, S., 2007. The SemEval-2007 WePS Evaluation: Establishing a Benchmark for the Web People Search Task. In Proceedings of the Fourth International Workshop on Semantic Evaluations, 64-69.

Ueda, H., Murakami, H., and Tatsumi, S., 2009. A ssigning Vocation-Related Information to Person Clusters for Web People Search Results. In Proceedings of the 2009 Global Congress on Intelligent Systems (GCIS 2009), 4, 248-253.

Murakami, H., Takamori, Y., Ueda, H., and Tatsumi, S., 2009. Assigning Location Information to Display Individuals on a Map for Web People Search Results. In Proceedings of The Fifth Asia Information Retrieval Symposium (AIRS 2009), 26-37.

Wan, X., Gao, J., Li, M., Ding, B., 2005. Person resolution in person search results: WebHawk. In CIKM2005, Proceedings of the Fourteenth ACM Conference on Information and Knowledge Management, 163-170.

Matsuo, Y., Mori, J., Hamasaki, M., Ishida, K., Nishimura, T., Takeda, H., Hashida, K., Ishiduka, M., 2006. Polyphonet: An advanced social network extraction system, In $W W W 2006,397-406$.

Mori, J., Basselin, A. Kroner, and A. Jameson, 2008. Find me if you can: Disigning Interfaces for People Search, In Proceedings of the 13th international conference on Intelligent user interfaces, 377-380. 